\title{
Cluster Based Feedback Provision Strategies in Intelligent Tutoring Systems
}

\author{
Sebastian Gross ${ }^{1}$, Xibin Zhu², Barbara Hammer ${ }^{2}$, and Niels Pinkwart ${ }^{1}$ \\ 1 Clausthal University of Technology, Germany \\ \{sebastian.gross, niels.pinkwart\}@tu-clausthal.de \\ 2 Bielefeld University, Germany \\ \{xzhu, bhammer\}@techfak.uni-bielefeld.de
}

\begin{abstract}
In this paper, we propose the use of machine learning techniques operating on sets of student solutions in order to automatically infer structure on these spaces. Feedback opportunities can then be derived from the clustered data. A validation of the approach based on data from a programming course confirmed the feasibility of the approach.
\end{abstract}

Keywords: intelligent tutoring systems, ill-defined domains, machine learning.

\section{Introduction}

In many domains such as law, argumentation or art, most problems are illdefined and have ambiguous solutions that can be argued for (and against!) but that are impossible to verify formally [4. If ITSs cannot rely on explicit models, it may be possible to acquire information about the domain in terms of examples given by students or experts. Since these learnt models are widely data driven, machine learning techniques such as clustering constitute a key technology to infer meaningful information from given examples. The approach presented in this paper is based on clusters of student solutions where the solutions within each cluster might have a different quality but are structurally similar.

\section{Clustered Solution Spaces: Feedback Strategies}

In this section, we discuss two cases of how feedback based on clustered sets of student solutions can be given in the absence of formal domain models.

In the first case, we assume that grades for most of the student solutions in the data set are available (e.g., via assessments by human tutors). Every class of the solution space can then be represented by one student solution which has a high structural similarity to the other student solutions in the class (i.e., it is near the center of the class), and has a high grade (i.e., it is a good solution).

These representative solutions can then be used to give feedback to students who submit a new solution. A newly submitted (potentially erroneous) student 
solution will then be analyzed (in terms of which class it belongs to) and compared structurally to the representative solution within this class. The result of this comparison can be fed back to students in various forms, including (i) a direct comparison showing the student's solution and the representative solution, highlighting differences between both, or (ii) the highlighting of potentially erroneous parts in the student's solution (i.e., the parts where it differs from the representative solution) without explicitly showing the representative solution.

In the second case, we assume that reliable scores for solutions are not available. As such, representative good solutions as previously defined cannot be computed. Here, one option is to use peer reviewing among the group of students. Another way of providing feedback is peer tutoring [3] in which a reviewing student is tutoring another student. The peer tutor can give hints about evident mistakes and can ask questions about potential mistakes. In this second case, the clustering can be helpful for selecting appropriate peer reviewers or tutors.

\section{Validating and Discussing the Approach: a Case Study}

To validate our approach and investigate whether our method for feedback provision is practically applicable, we conducted a case study. We used a data set from a Java programming class. For this data, scores assigned by human experts were available for every student solution. The solution clusters were computed using affinity propagation (AP) 2. Similarities between solutions were computed based on Plaggie [1], a plagiarism detection algorithm that calculates a simple structural comparison of two programs. This way, we represented the space of all solution structures by means of a small number of prototypical correct solutions (case 1 from above). In general, this test confirmed our expectations: the resulting clusters were relatively clear, and overall the ways of feedback provision as suggested above made sense for this data set.

However, there were also some limitations. For very poor student solutions that lack any structure, the methods still have drawbacks - these were added to one of the clusters, but the ways of feedback provision did not make much sense. As long as the structural similarity between solutions is high, our methods for feedback provision make sense - less so if elements within a cluster are dissimilar.

\section{References}

[1] Ahtiainen, A., Surakka, S., Rahikainen, M.: Plaggie: Gnu-licensed source code plagiarism detection engine for java exercises. In: Proceedings of the 6th Baltic Sea Conference on Computing Education Research: Koli Calling 2006, Baltic Sea 2006, pp. 141-142. ACM, New York (2006)

[2] Frey, B.J., Dueck, D.: Clustering by passing messages between data points. Science 315, 972-976 (2007)

[3] Goodlad, S., Hirst, B.: Peer tutoring: a guide to learning by teaching. Kogan Page (1989)

[4] Lynch, C., Ashley, K.D., Pinkwart, N., Aleven, V.: Concepts, structures, and goals: Redefining ill-definedness. International Journal of Artificial Intelligence in Education 19(3), 253-266 (2010) 